\title{
Une comparaison préliminaire de modèles rhéologiques pour l'argile plastique : l'exercice communautaire INTERCLAY (phase pilote-1989)
}

\section{A preliminary comparison of rheological models for plastic clay: the community project INTERCLAY (pilot phase-1989)}

\author{
B. CÔME
}

Commission des Communautés Européennes

Rev. Franç. Géotech. n 55 , pp. $75-80$ (avril 1991)

\section{Résumé}

On a comparé des modèles décrivant le comportement différé de l'argile plastique de Boom (Belgique), selon deux approches: (a) fluage, et (b) dissipation de pression interstitielle. Dans une première phase pilote, trois équipes ont résolu, indépendamment, deux problèmes simplifiés : (1) l'excavation d'un tunnel profond avec pose d'un revêtement rigide et perméable ; (2) un essai de fluage in situ au dilatomètre. Les données provenaient d'essais de laboratoire sur le même matériau. On présente ici les premiers enseignements tirés de cette comparaison.

Ces travaux ont été réalisés dans le cadre du $3^{\circ}$ programme communautaire de $R \& D$ sur " la gestion et le stockage des déchets radioactifs".

\footnotetext{
Abstract

Two kinds of models for describing the time-dependent behaviour of plastic Boom clay (Belgium) were compared: (a) creep model, and (b) pore pressure dissipation model. In a first exploratory phase, three teams solved, independently, two idealized problems : (1) the excavation of a deep tunnel with a stiff and permeable lining; (2) an in situ dilatometer creep test. Data were obtained from laboratory tests on the same material. The first lessons drawn from this comparison are presented.

This work was carried out in the framework of the 3 rd Community R\&D programme on "management and storage of radioactive waste".
} 


\section{INTRODUCTION}

Pour décrire le comportement différé des argiles plastiques, on a ordinairement recours à deux approches. L'une considère le matériau comme un solide monophasique susceptible de se déformer par fluage : c'est l'approche de la mécanique des roches. L'autre considère le rôle de l'eau interstitielle incluse dans les pores du matériau, et identifie, pour cause essentielle des déformations, une redistribution des pressions interstitielles sous l'effet des forces appliquées : cette description est du domaine de la mécanique des sols, Certains matériaux, tels l'argile plastique de Boom (Belgique), sont à la frontière entre un sol rigide et une roche tendre, et peuvent donc a priori être étudiés par l'une ou l'autre de ces méthodes.

Comme l'argile de Boom est un matériau candidat pour l'évacuation de déchets radioactifs ( $B O N N E$, 1987), il est nécessaire de pouvoir prédire de façon fiable les perturbations mécaniques et/ou hydrauliques entraînées dans ce matériau par la création d'un dépôt, et la mise en place de déchets. C'est dans ce contexte qu'une comparaison entre les deux approches mentionnées plus haut prend tout son sens, chacune prétendant rendre compte de façon satisfaisante de l'évolution du matériau sous l'effet des diverses sollicitations.

Une telle comparaison apparaît d'autant plus aisée que l'argile de Boom, étudiée en particulier par le Centre d'Etude de l'Energie Nucléaire (CEN/SCK) à Mol depuis 1975 , est probablement l'un des rares matériaux sur lequel on ait réalisé un aussi grand nombre d'essais géomécaniques, de laboratoires et en place, incluant en particulier des essais de fluage, et aussi des essais triaxiaux drainés et cedométriques. Les éléments nécessaires pour une comparaison systématique des deux « philosophies» évoquées sont donc réunis: une première tentative avait d'ailleurs été esquissée dès 1987 en ce sens (DE BRUYN, 1989). Dans un esprit légèrement différent, davantage orienté vers des modèles dérivés d'essais de laboratoire sur l'argile de Boom, la Commission des Communautés Européennes a lancé, en 1989, la phase pilote d'un projet dénommé INTERCLAY, devant déboucher, à terme, sur une évaluation rigoureuse des deux approches, "fluage s et " pression interstitielle».

Ont contribué à cette phase pilote :

- le CEN/SCK, opérateur du laboratoire souterrain à Mol, et coordonnateur du programme de recherche sur le matériau;

- le Laboratoire de Mécanique des Solides (LMS)

à Palaiseau associé au CEA-ANDRA (F) ;

- l'ISMES, Bergame (I) :

- le Geotechnical Consulting Group (GCG), Londres, associé à la City University, avec lappui du Building Research Establishment (GB).

\section{DONNÉES CONCERNANT LE MATÉRIAU}

En 1984, des blocs d'argile avaient été soigneusement prélevés dans une petite galerie (profondeur : $240 \mathrm{~m}$ ) du laboratoire souterrain sous le site du CEN/SCK à $\mathrm{Mol}$, et répartis entre plusieurs laboratoires.

Au LMS furent réalisés des essais de compression non drainés, de fluage mono et triaxial, et de fluage sur tube creux, d'où fut dérivé un modèle de comportement élasto-visco-plastique avec radoucissement décrivant la déformation de l'argile de Boom en terme de fluage (ROUSSET, 1988).

De leur côté, ISMES et GCG adoptèrent une description du matériau en terme de dissipation de pression interstitielle, couplant :

- la loi de Darcy pour l'écoulement de l'eau interstitielle ;

- l'équation de continuité de l'ensemble eau-pores ; - une loi de comportement, en contraintes effectives, du squelette solide. Pour l'exercice INTERCLAY, GCG et ISMES utilisèrent un modèle « CAM-CLAY modifié „(BALDI, 1987 ; HORSEMAN, 1987).

On notera que les résultats d'essais, tels que courbes cedométriques, peuvent faire l'objet d'interprétations légèrement différentes ; c'est ainsi que les grandeurs caractéristiques du matériau ne furent pas identiques pour GCG et ISMES (par exemple la perméabilité fut prise égale respectivement à $4.10^{-12}$ et $10^{-12} \mathrm{~m} / \mathrm{s}$ par ces équipes).

\section{PROBLÈMES CONSIDÉRÉS}

Pour garder une taille raisonnable à l'exercice, on n'a considéré que des problèmes théoriques et simplifiés, mais cependant pas trop différents de situations réelles d'essais conduits à environ $220 \mathrm{~m}$ de profondeur dans l'argile de Boom (CÔME, 1989). En termes généraux, ces problèmes se ramènent au comportement de tubes très épais, modélisables en symétrie de révolution et en déformations planes; les chargements et le matériau sont supposés isotropes. Enfin, les conditions initiales et aux limites sont mathématiquement bien spécifiées, ce qui n'est pas toujours le cas dans la réalité d'une excavation souterraine.

Les problèmes proposés, et résumés ci-dessous, firent l'objet de spécifications préparées par la Commission. tenant compte des commentaires exprimés par les participants.

\subsection{Problème " excavation de tunnel profond "}

Dans un cylindre d'argile de rayon $50 \mathrm{~m}$ chargé par 4,4 $\mathrm{MPa}$ de pression totale, dont $2.2 \mathrm{MPa}$ de pression interstitielle, un tunnel coaxial de rayon $2,5 \mathrm{~m}$ est « excavé » (à $220 \mathrm{~m}$ de profondeur), en faisant décroître la pression totale intérieure de $4,4 \mathrm{MPa}$, valeur initiale, à $1 \mathrm{MPa}$, en $10^{6}$ secondes (environ 12 jours). A cette date, le déplacement radial est bloqué par un revêtement infiniment rigide et perméable. On demanda l'évolution de la contrainte totale sur le revêtement, et à certains points dans le massif. ainsi que l'évolution de la pression interstitielle en ces mêmes points, jusqu'à $10^{9}$ secondes (environ 32 ans). Les équipes utilisant l'approche « pression interstitielle " durent justifier en outre l'évolution de cette pression en paroi d'excavation, jusqu'à ce qu'elle reste à une valeur nulle (condition de drainage par le tunnel). 


\subsection{Problème « essai in situ de fluage au dilatomètre »}

On envisage une membrane cylindrique de rayon 7.5 $\mathrm{cm}$, logée à $220 \mathrm{~m}$ de profondeur dans un tube coaxial d'argile épais de rayon $1,5 \mathrm{~m}$, chargé par 4,4 $\mathrm{MPa}$ de contrainte totale, dont $2,2 \mathrm{MPa}$ de pression interstitielle. La membrane initialement gonflée à 4,4 $\mathrm{MPa}$ est progressivement dégonflée jusqu'à $2 \mathrm{MPa}$ en 1 heure $\left(3,6 \cdot 10^{3} \mathrm{~s}\right)$; la pression interne reste maintenue ensuite à $2 \mathrm{MPa}$. On demanda l'évolution de la convergence de la membrane au cours du temps, ainsi que celle des contraintes totales et pressions interstitielles dans le tube. L'imperméabilité de la membrane correspond à une condition mathématique de flux d'eau constamment nul.

\section{RÉSULTATS}

Les calculs de contraintes et déplacements ont été réalisés par les diverses équipes selon l'arrangement du tableau suivant. Les trois équipes ont résolu les problèmes indépendamment l'une de l'autre, les résultats (inconnus à l'avance des participants) étant rassemblés par la Commission pour être présentés sous forme de diagrammes comparatifs par le CEN/SCK.

Equipe

Approche

Code de calcul

LMS

GCG

ISMES

\section{GEOMEC CRISP}

Le détail des résultats est donné dans le rapport de synthèse du projet (CCE, 1990). A titre d'exemple, les figures 1 à 4 donnent respectivement l'évolution:

- de la pression totale sur le revêtement,

- de la pression interstitielle à différentes distances dans le massif,

dans le cas du calcul du tunnel ;

- de la convergence de la paroi du forage,

- de la contrainte totale radiale.

dans le cas du calcul de l'essai au dilatomètre.

\section{DISCUSSION DES RÉSULTATS}

\subsection{Constatations}

Bien que les courbes calculées par les différentes équipes ne coïncident pas entièrement, on peut cependant tout d'abord noter une certaine similitude d'allure, non seulement dans les tendances générales, mais aussi dans les ordres de grandeur calculés : la variation des résultats, du plus faible au plus grand, est du simple au double en général. Il est assez remarquable que des philosophies de modélisation aussi différentes que celles considérées ici fournissent des résultats prévisionnels somme toute assez bien groupés.

Bien que - on l'a souligné plus haut - les problèmes ne soient que des simplifications de cas réels, on peut néanmoins tenter une comparaison avec des

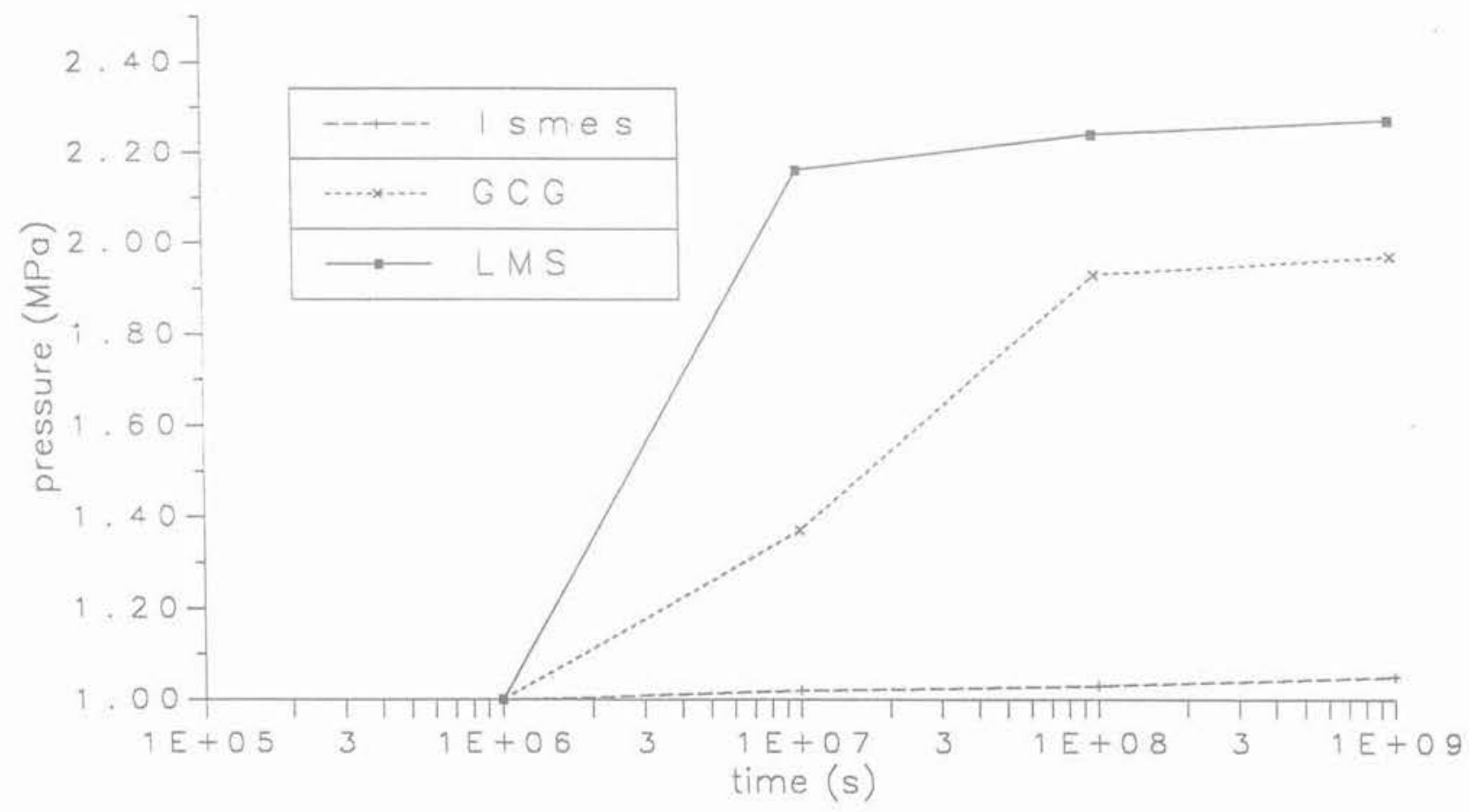

Fig. 1. - Excavation du tunnel: calcul de la pression totale sur le revêtement.

Fig. 1. - Tunnel excavation: total pressure on the lining (MPa). 


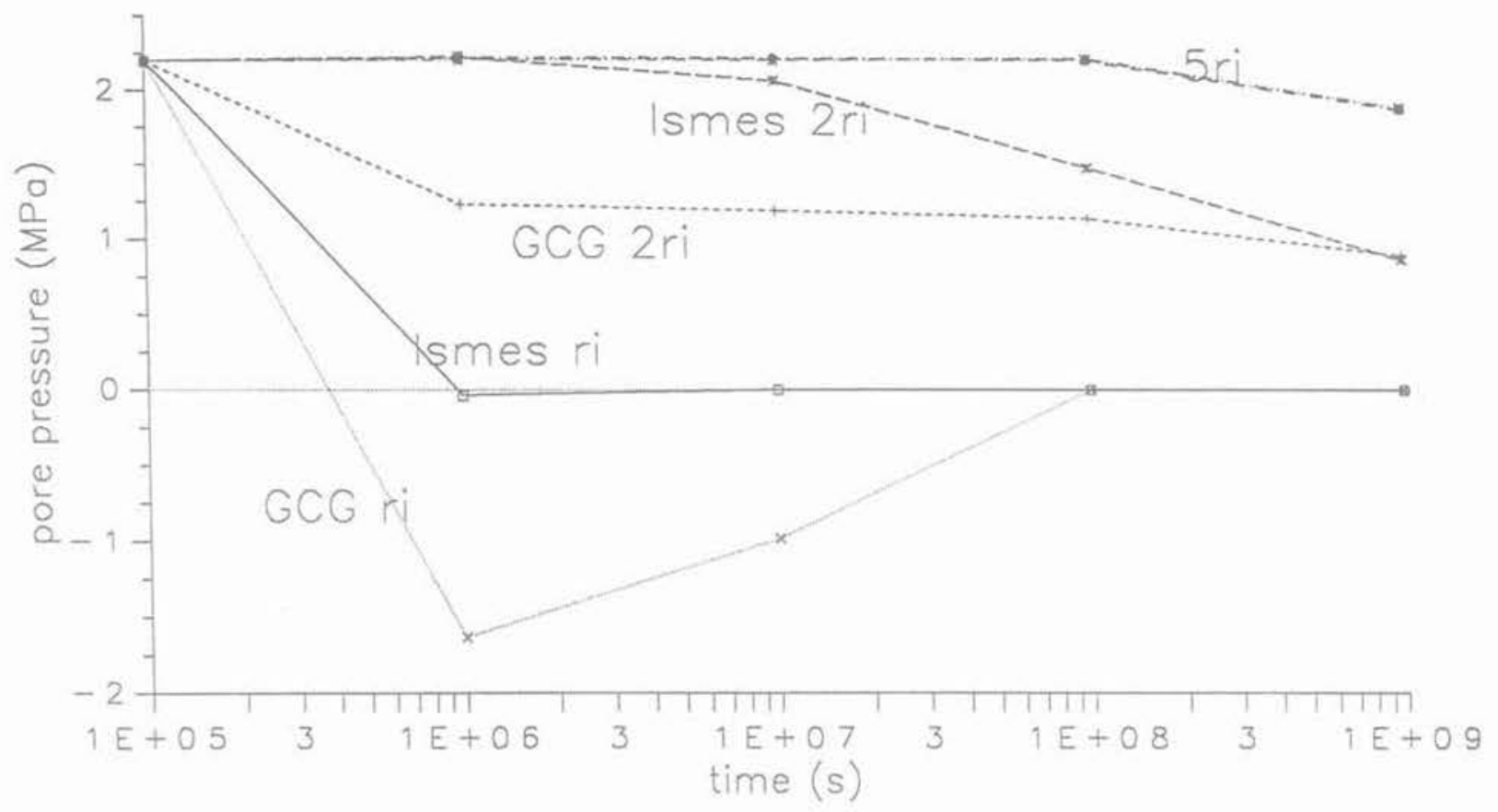

Fig. 2. - Excavation du tunnel: calcul de la répartition des pressions interstitielles à 1,2 et 5 rayons de cavité.

Fig. 2. - Tunnel excavation: pore pressure (MPa) at ri (gallery wall), $2 \mathrm{ri}, 5 \mathrm{ri}$

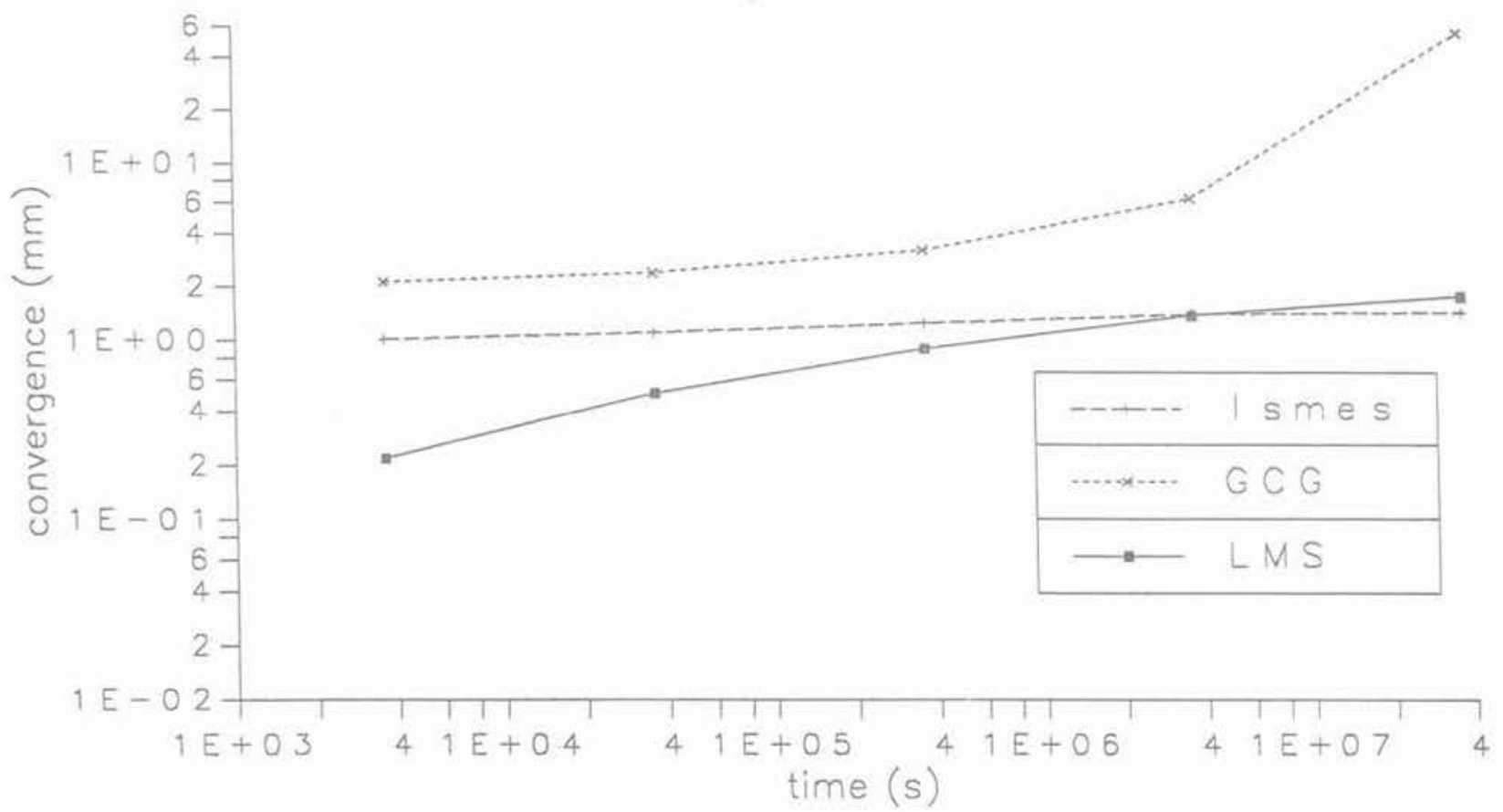

Fig. 3. - Essai au dilatomètre : calcul de la convergence de la paroi au cours du temps.

Fig. 3. - Dilatometer test : displacement function of time, radius $=0,075 \mathrm{~m}$. 


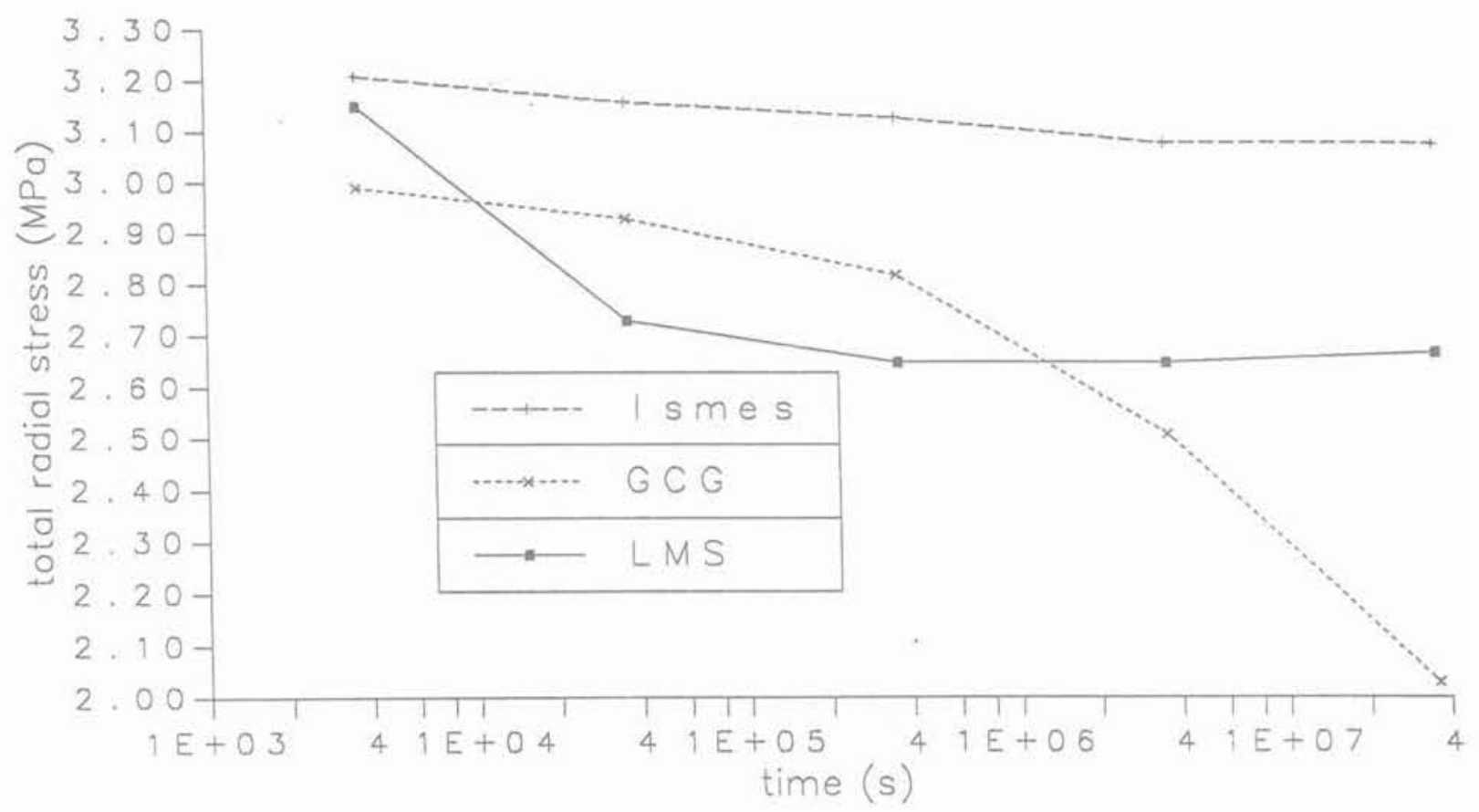

Fig. 4. - Essai au dilatomètre: évolution calculée de la contrainte totale radiale dans le massif, à 1,5 rayon de cavité.

Fig. 4. - Dilatometer test : total radial stress function of time at $r=1.5 \mathrm{ri}$.

mesures obtenues dans des expériences réelles dans l'installation souterraine de Mol (CÔME, 1989). A titre d'exemple, la pression totale mesurée sur le revêtement d'un tronçon de galerie (à revêtement certes plus déformable que dans le problème) est de l'ordre. de 1,5 $\mathrm{MPa}, 2$ ans après l'excavation, et varie peu depuis cette date; l'accord est acceptable avec les résultats calculés. D'autre part, un essai de fluage au dilatomèive dans un forage du laboratoire souterrain a relevé une convergence relative « différée », sous $2 \mathrm{MPa}$ de pression intérieure, comprise entre $1.5 \mathrm{et}$ $2 \%$, ce qui n'est pas très différent des valeurs calculées après 10000 heures, soit 400 jours. Dans une perspective de génie civil " ordinaire , on peut donc considérer que les modèles rhéologiques utilisés ici pour des calculs prévisionnels, prenant pour grandeurs d'entrée les résultats de mesures sur échantillons, remplissent leurs objectifs, et sont donc utilisables avec un degré de fiabilité suffisant.

Deux constatations supplémentaires viennent cependant tempérer cet optimisme: d'abord, l'absence d'unanimité entre les prévisions (donc entre les modèles), même pour l'approche "pression interstitielle», où les grandeurs d'entrée sont finalement peu différentes ; et, aussi, le désaccord entre les calculs des pressions interstitielles et les valeurs mesurées en place autour du laboratoire souterrain de Mol dans des conditions relativement voisines.

On dispose en effet de mesures par mini-piézomètre, selon lesquelles l'excavation d'un tunnel (appelé $\alpha$ Test Drift ») a fait immédiatement chuter la pression interstitielle d'au moins $1 \mathrm{MPa}$, même à 5 rayons de tunnel de la paroi ; les calculs INTERCLAY montrent qu'il faut plusieurs mois pour que la pression intersti- tielle commence à être influencée par l'excavation. Compte tenu de l'importance de tout ce qui touche au mouvement de l'eau souterraine pour la sûreté de l'évacuation de déchets en formations argileuses, on conçoit qu'il s'agit ici d'un point clé, ainsi mis en évidence par l'exercice ; il sera d'ailleurs repris ci-dessous.

\subsection{Limites de l'exercice et précautions à prendre}

La taille réduite de l'exercice a certes permis d'obtenir rapidement les intéressantes conclusions que l'on vient de tracer brièvement; elle constitue cependant un inconvénient notable quant à l'appréciation complète de ces résultats. En effet, vu le petit nombre de calculs finalement réalisés, il n'est pas possible d'évaluer le poids respectif de plusieurs facteurs importants pour expliquer les différences observées. On ne peut ainsi attribuer avec certitude un rôle déterminant :

- aux modèles rhéologiques eux-mêmes ;

- ou aux grandeurs d'entrée utilisées dans ces modèles ;

- ou, enfin, aux aspects purement numériques des solutions tels que densité du maillage d'éléments finis, schémas d'intégration, etc.

Qui plus est, le fait que ces problèmes soient des schématisations de situations réelles, et non leur réplique fidèle, interdit de qualifier une solution comme «correcte » ou \& fausse », ce qui constitue une limitation supplémentaire quant à l'appréciation des résultats et - en définitive - quant à la démonstration de l'adéquation réelle des modèles rhéologiques considérés. 
Il n'en demeure pas moins clair que, si l'on souhaite prédire de façon satisfaisante le régime d'écoulerment de l'eau autour de galeries excavées dans des milieux sédimentaires à très faible perméabilité, il faudra disposer de modèles couplant la réponse mécanique du squelette et celle de l'eau interstitielle.

\section{CONCLUSIONS}

L'intérêt de cette phase-pilote d'INTERCLAY réside essentiellement dans les questions soulevées, beaucoup plus que dans les résultats calculés eux-mêmes. A ce titre, il serait souhaitable de tenir compte des aspects positifs, et aussi des lacunes, de cette phase préliminaire, pour définir un exercice plus vaste (et plus rigoureux) d'intercomparaison systématique des modèles rhéologiques, des données et des outils numériques (codes de calcul) utilisables pour le calcul prévisionnel en matière de géomécanique des argiles plastiques très peu perméables. Un tel exercice comparatif pourrait s'inscrire dans le prochain programme de la Communauté sur ce sujet.

\section{REMERCIEMENTS}

Les participants au projet pilote INTERCLAY ont tous apporté, avec enthousiasme, leurs contributions scientifiques et/ou financières : qu'ils en soient ici chaleureusement remerciés.

\section{BIBLIOGRAPHIE}

BALDI G, et al. (1987). Calibration of mathematical models for simulation of thermal, seepage and mechanical behaviour of Boom clay. Rapport CCE $n^{\circ}$ EUR 10924, Luxembourg.

BONNE A. (ed.) (1987), R\&D programme on radioactive waste disposal into geological formations : study of a clay formation. Rapport CCE $n^{\circ}$ EUR 11205, Luxembourg.

CCE (1990), The CEC benchmark INTERCLAY Results of pilot phase (January/June 1989) Rapport CCE n EUR 12791, Luxembourg.

CÔME B. (ed.) (1989), Geomechanics of clay with a view to radioactive waste disposal. Comptes rendus d'une session technique, Bruxelles, Décembre 1988. Rapport CCE n EUR 12027 Luxembourg.

DE BRUYN D., AUBRY D., ROUSSET G. (1989) Comparison of rheological models in view of pre. dicting the behaviour of a deep clay host rock during the construction of a radwaste repository. Proceeding NUMOG 89, Elsevier Publishers, pp. 724-773.

HORSEMAN S.T. et al. (1987), Geotechnical characterisation of Boom clay in relation to the dis. posal of radioactive waste. Rapport CCE $n^{\circ}$ EUR 10987, Luxembourg.

ROUSSET G. (1988), „Comportement mécanique des argiles profondes. Application au stockage des déchets radioactifs $\%$. Thèse de doctorat ENPC, Ecole Polytechnique, Palaiseau (F). 